\title{
ALLOGRAFT VERSUS AUTOGRAFT BONE IN SCOLIOSIS SURGERY
}

\author{
C. A. F. DODD, C. M. FERGUSSON, L. FREEDMAN, G. R. HOUGHTON, D. THOMAS
}

From the Nuffield Orthopaedic Centre, Oxford

\begin{abstract}
The results of a study of the use of autograft versus allograft bone in the surgery of idiopathic adolescent scoliosis are presented. Two groups of patients, matched for age, sex, level and angle of curve, received bone grafts, 20 patients having autogenous bone from the iliac crest and the other 20 having donor bone from a bone bank. Both groups had otherwise identical posterior fusions and Harrington instrumentation.

There was no difference between the two groups in a blind, radiographic assessment of bone graft mass at six months, nor in maintenance of the curve correction over the same period. No major operative complications nor failures of instrumentation were encountered. There was, however, a marked reduction in operative time and blood loss in the patients receiving donor bone and also a much lower incidence of late symptoms relating to the operative sites. We conclude that, even in the presence of adequate iliac crest, the use of bank bone is superior for grafting in idiopathic scoliosis surgery.
\end{abstract}

Spinal instrumentation and bone grafting have an established role in the treatment of idiopathic scoliosis and its success lies in good initial correction, sound fixation of the implant and subsequent incorporation of the graft to achieve solid fusion for long-term stabilisation (Goldstein 1969).

Although autograft bone has traditionally been considered as the ideal in spinal fusion, it is not without its complications (Friedlaender 1982). These include problems at the donor site such as infection (Goldstein 1969) and increased blood loss and operative time (Malinin and Brown 1981). There may also be limitations because of inadequate quality (Cloward 1980), or insufficient quantity as in the patient with neuromuscular scoliosis who has deficient iliac crests (McCarthy et al. 1986). These problems have encouraged surgeons to look for other sources.

C. A. F. Dodd, FRCS, Senior Registrar

Accident Service, John Radcliffe Hospital, Headington, Oxford OX3 9DU, England.

C. M. Fergusson, FRCS, Senior Orthopaedic Registrar

G. R. Houghton, FRCS, Consultant Orthopaedic Surgeon

D. Thomas, P.Dip.Stat, Research Statistician

Oxford Orthopaedic Engineering Centre, Nuffield Orthopaedic Centre, Headington, Oxford OX3 7LD, England.

L. Freedman, FRCS, Senior Orthopaedic Registrar

Prince of Wales Orthopaedic Hospital, Rhydlafar, Cardiff CF5 6XG, Wales.

(C) 1988 British Editorial Society of Bone and Joint Surgery

$0301-620 \mathrm{X} / 88 / 3068 \$ 2.00$

J Bone Joint Surg [Br] 1988;70-B:431-4.
The use of allograft bone provides a reasonable alternative to meet the growing need for primary or supplementary graft material. Allograft bone has been used for many years for a variety of orthopaedic operations; the problems of associated transfer of infection (Tomford, Ploetz and Mankin 1986) and antigenicity (Langer et al. 1975; Friedlaender, Strong and Seli 1976; Johnson, Brown and Lasky 1985) have been widely studied and represent a minimal risk to the patient. The use of allografts from femoral heads has recently been receiving more attention (Malinin, Rosomoff and Sutton 1977; Malinin and Brown 1981); guidelines for its collection, its suitability for graft material, and its storage are now established (Tomford et al. 1986).

Confidence in femoral head bone for allografts and its success in establishing satisfactory fusion without complications, strongly favours its routine use as preferred graft material even in the presence of available autogenous bone. The work of Aurori and Kumar supports this view (Aurori et al. 1984). We have therefore conducted a prospective trial to assess the use of femoral head allograft versus autograft bone in idiopathic scoliosis surgery.

\section{PATIENTS AND METHODS}

Allograft bone was introduced into scoliosis surgery in Oxford in January 1984. This study comprised a consecutive series of 20 patients who underwent pos- 
terior fusion and Harrington rod instrumentation with autogenous graft from July 1982 to December 1983 (Group A) and the first 20 patients who had donor bone (Group B). The notes and radiographs of these 40 patients form the basis of this study; in each case the diagnosis was idiopathic adolescent scoliosis.

Surgical technique. Posterior spinal fusion was performed by one surgeon (GRH). Subperiosteal exposure of the posterior vertebral surfaces to be fused was undertaken from the laminae to the tips of the transverse processes. After facet joint excision and thorough decortication, donor bone was laid generously and packed onto the prepared surfaces. Correction of the curves was maintained by Harrington rod instrumentation using a bifid hook superiorly and a Leatherman hook inferiorly together with a four-hook apical compression system and two transverse loading wires. The patients were all treated with a short course of postoperative cephalosporins and nursed supine until suture removal at two weeks. They were then mobilised in plaster in a position of elongation, derotation and flexion (EDF cast) for six months.

The allografts were obtained from femoral heads that had been harvested at the time of reconstructive hip procedures. Only bone from suitable donors who had fulfilled the recommendations of Friedlaender and Mankin (1981) were considered. Routinely a small piece of bone was sent for culture and donor blood was taken for Australian antigen, HIV estimation, and routine syphilis screening. All bone was washed with saline and stored at $-21^{\circ} \mathrm{C}$. Most bone was used within three months.

Assessment. A radiographic assessment was made of the bone graft at six months. Two of the authors (CAFD and CMF) each reviewed all the films independently and "blind" to the patient's name and group. The amount of graft was classified as little, moderate, or large. A questionnaire was sent to each patient asking specifically whether they had symptoms related to either the donor site scar or to the mid-line scar posteriorly. They were also asked whether they were pleased with the result.

Correction of the curve. This is perhaps the important measure, the other comparisons mainly testing whether or not the two groups were matched. Correction was defined as the operative correction of the Cobb angle, divided by the initial Cobb angle, and expressed as a percentage. It was thus standardised and independent of the initial Cobb angle.

Statistical analysis. Student's $t$-tests were used to compare pairs of means for the following variables: age of patient; total blood loss during the operation (millilitres); duration of operation (minutes); the Cobb angle initially, postoperatively and six months after surgery; and fusion length as a percentage of total spinal length (considering vertebra from $\mathrm{T} 1$ to $\mathrm{L} 5$, and assuming no patient had a sixth lumbar vertebra).

\section{RESULTS}

Assessment of graft revealed that in Group A (autografts) there were eight Type 1 (small) graft masses, six Type 2 (medium) masses, and six Type 3 (large) masses. In Group B (allografts) there were nine Type 1 masses, six Type 2 masses and five Type 3 masses.

All questionnaires except two, one from each group, were returned. Eight out of the 20 in Group A specifically mentioned persisting pain in the oblique donor scar site; two had patchy paraesthesia in relation to the graft scar which persisted for at least six months. No patient complained of postoperative backache.

Table I shows the statistical details for all the variables measured and Figures 1 and 2 are histograms showing total blood loss and operation times in each group.

\section{Complications}

One patient in the allograft group had infection at the femoral pin site following pre-operative traction. Otherwise, there were no recorded cases of infection, either superficial or deep. In the allograft group, one wound oozed for three days but was sterile on culture. Four patients had complications directly attributable to the EDF cast: there was one case of ileus in the autograft group, one iliac vein thrombosis, one cast syndrome which responded to conservative treatment and one meralgia paraesthetica in the allograft group. One patient who had had pre-operative traction had stiff knees postoperatively which required manipulation

Table I. Statistical details of all variables measured in Group A (autografts) and Group B (allografts)

\begin{tabular}{|c|c|c|c|c|c|c|}
\hline Variable & Group & Mean & s.e. & t-value & Probability & \\
\hline Age & $\begin{array}{l}\mathbf{A} \\
\mathbf{B}\end{array}$ & $\begin{array}{l}15.1 \\
14.6\end{array}$ & $\begin{array}{l}0.34 \\
0.35\end{array}$ & 1.05 & $\mathrm{p}>0.30$ & NS \\
\hline $\begin{array}{l}\text { Operation time } \\
\text { (minutes) }\end{array}$ & $\begin{array}{l}\mathbf{A} \\
\mathbf{B}\end{array}$ & $\begin{array}{l}176.0 \\
140.6\end{array}$ & $\begin{array}{l}4.48 \\
4.77\end{array}$ & 5.41 & $\mathrm{p}<0.001$ & 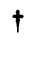 \\
\hline Total blood loss (ml) & $\begin{array}{l}\mathbf{A} \\
\mathbf{B}\end{array}$ & $\begin{array}{l}1735.0 \\
1536.3\end{array}$ & $\begin{array}{l}74.20 \\
81.01\end{array}$ & 1.83 & $p>0.07$ & NS \\
\hline Fusion length & $\begin{array}{l}\mathbf{A} \\
\mathbf{B}\end{array}$ & $\begin{array}{l}7.7 \\
8.0\end{array}$ & $\begin{array}{l}0.21 \\
0.31\end{array}$ & & & \\
\hline Fusion length $\%$ & $\begin{array}{l}\mathbf{A} \\
\mathbf{B}\end{array}$ & $\begin{array}{l}45.3 \\
47.1\end{array}$ & $\begin{array}{l}1.22 \\
1.81\end{array}$ & -0.81 & $\mathrm{p}>0.42$ & NS \\
\hline \multicolumn{7}{|l|}{ Cobb angle (degrees) } \\
\hline Pre-operative & $\begin{array}{l}\mathbf{A} \\
\mathbf{B}\end{array}$ & $\begin{array}{l}60.4 \\
58.8\end{array}$ & $\begin{array}{l}2.93 \\
3.26\end{array}$ & 0.38 & $p>0.71$ & NS \\
\hline Postoperative & $\begin{array}{l}\mathbf{A} \\
\mathbf{B}\end{array}$ & $\begin{array}{l}26.5 \\
31.5\end{array}$ & $\begin{array}{l}2.34 \\
2.44\end{array}$ & -1.47 & $p>0.15$ & NS \\
\hline At six months & $\begin{array}{l}\mathbf{A} \\
\mathbf{B}\end{array}$ & $\begin{array}{l}29.5 \\
32.5\end{array}$ & $\begin{array}{l}2.01 \\
2.44\end{array}$ & -0.96 & $\mathrm{p}>0.34$ & NS \\
\hline Correction & $\begin{array}{l}\mathbf{A} \\
\mathbf{B}\end{array}$ & $\begin{array}{l}57.1 \\
46.9\end{array}$ & $\begin{array}{l}2.37 \\
3.07\end{array}$ & -2.62 & $p>0.05$ & $*$ \\
\hline
\end{tabular}

* significant; † highly significant 

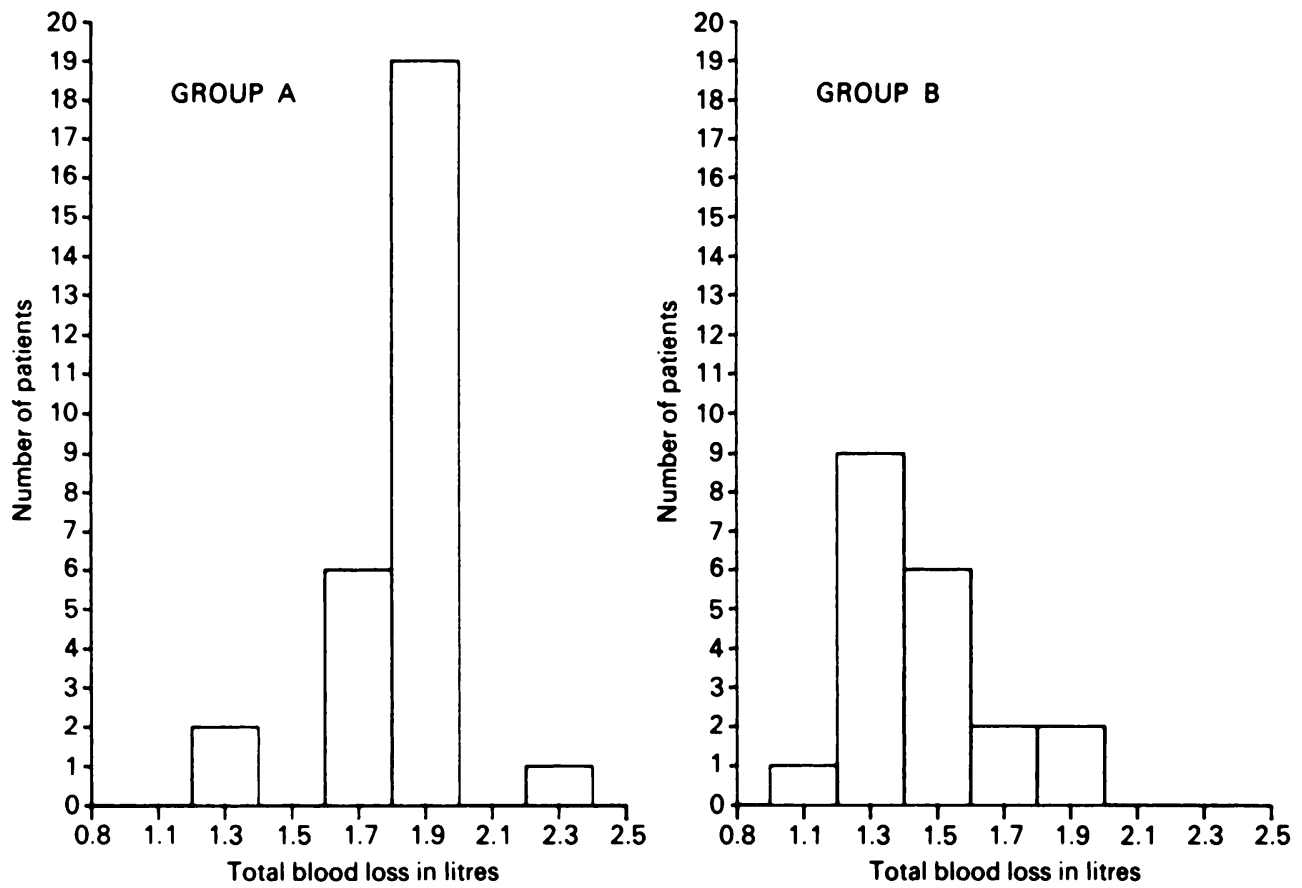

Fig. 1

Frequency histograms for total blood loss. The loss for Group A was greater than that for Group B though the difference was not significant at the 5\% level; it was significant at the $10 \%$ level.
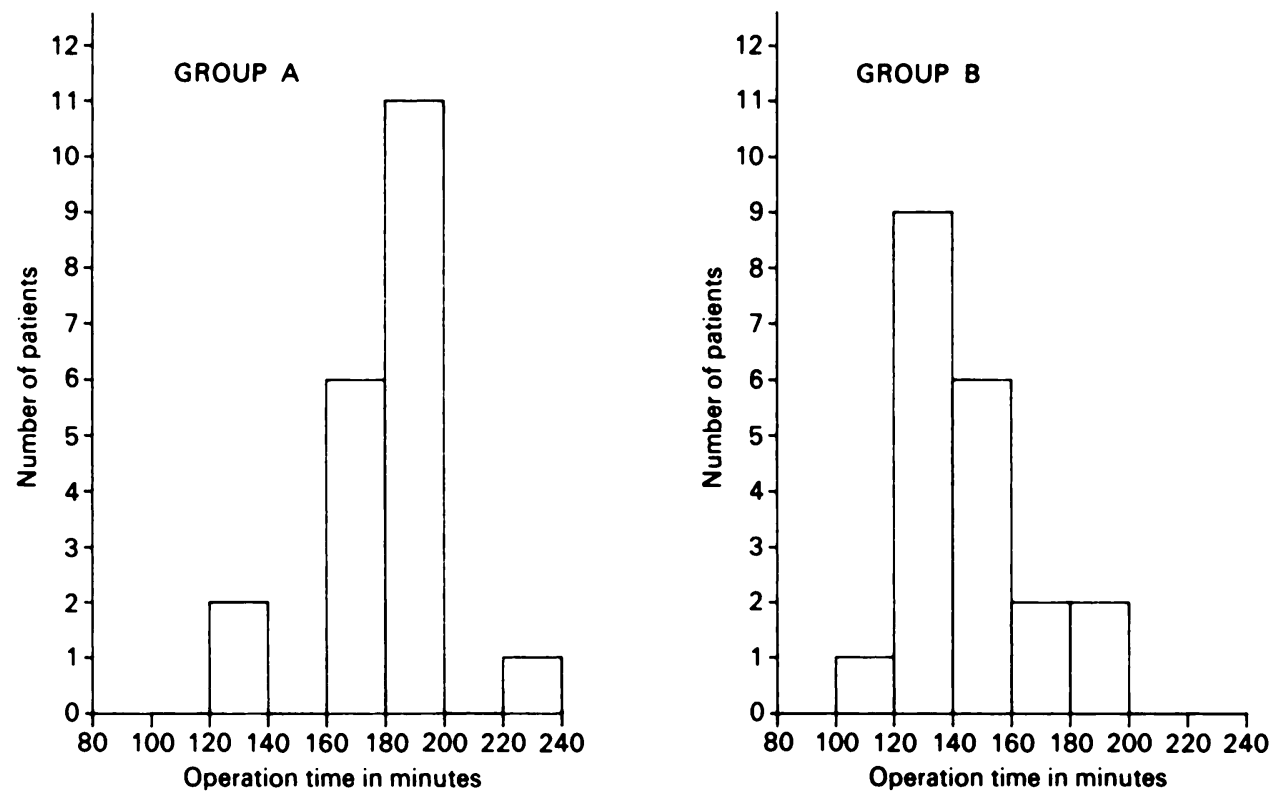

Fig. 2

Frequency histogram for operation times. Patients in Group A have significantly longer operating times than those in Group B, as indicated by the results of the $t$-test. 
under anaesthetic. There were no cases of pseudarthrosis and no implant migration, hook dislodgement or rod fracture in either group.

\section{DISCUSSION}

The use of allograft bone provides the only real alternative to autogenous graft in the quest for suitable material to secure predictable fusion in scoliosis surgery. It is usually used in the form of deep frozen or freezedried cadaveric graft or frozen graft taken from the femoral heads of patients undergoing hip replacement. The use of allografts from femoral heads has recently been receiving more attention (Malinin and Brown 1981; Friedlaender 1982) and with the regular performance of total hip arthroplasties and hemi-arthroplasties, femoral heads are readily available in most orthopaedic hospitals. As the bone is collected, stored and subsequently used in the same hospital, many of the financial and logistic implications of establishing a central bone bank are bypassed (Doppelt et al. 1981).

The infection and pseudarthrosis rate in our series compares favourably with those encountered in similar groups and is known to be less than in correction and fusion for paralytic and neuromuscular scoliosis. Reports are now appearing showing similar rates of fusion and infection after allografts and autografts in other areas of spinal surgery (Aurori et al. 1984). Structural failure has been reported in cervical fusion with allograft bone in children (Stabler et al. 1985). In our group there was an absence of implant failure which we attribute in part to the large, thick, fusion mass that was achieved with allograft bone which contributed significantly to a more stable, solid arthrodesis.

The results of the $t$-tests indicated that the only significant differences between the two groups were in operation time $(p<0.001)$ and correction $(p<0.05)$, both being greater in Group $A$. The percentage fusion lengths were not significantly different $(p>0.42)$. In neither group was the correlation between operation time and total blood loss significant at the $5 \%$ level; that is, a longer operation time did not necessarily imply a greater blood loss. The lower blood loss and operation time in the allograft group have obvious implications to any scoliosis unit.

Subjective assessment by the patient of postoperative discomfort confirms that the pain from iliac crest donor site is a real disadvantage of autogenous grafting: eight patients had pain, two of whom had patchy paraesthesia at the scar for six months. No patient complained of significant backache and objectively there was no significant difference in loss of correction at follow-up in either group. Simple radiographic assessment of fusion mass is a poor measure of quantity (Brown, Malinin and Davis 1976) but comparison could be made between the groups; within the limitations of the technique there were no differences. The fear that antigenicity might prove a major inhibitory problem appears unfounded. Several studies have shown that either freeze-drying or freezing bone to $-20^{\circ} \mathrm{C}$ significantly reduces its antigenicity. In our limited series, no direct problems could be attributed to the implantation of foreign bone.

Standard guidelines in the methods of bone banking are now widely accepted (Friedlaender and Mankin 1981) and we would recommend that allograft bone is the graft material of choice in spinal surgery. New government guidelines (Acheson 1987) since this trial have stressed the importance of getting consent from the donor before the bone is used, even if the bone is being removed for therapeutic purpose, as in total hip replacement.

No benefits in any form have been received or will be received from a commercial party related directly or indirectly to the subject of this article.

\section{REFERENCES}

Acheson D. DHSS circular 1987.

Aurori BF, Weierman RJ, Lowell HA, Nadel CI, Parsons JR. Pseudarthrosis after spinal fusion for scoliosis: a comparison of autogeneic and allogeneic bone grafts. Clin Orthop 1985;199:153-8.

Brown MD, Malinin TI, Davis PB. A roentgenographic evaluation of frozen allografts versus autografts in anterior cervical spinal fusions. Clin Orthop 1976;119:231-6.

Doppelt SH, Tomford WW, Lucas AD, Mankin HJ. Operational and financial aspects of a hospital bone bank. J Bone Joint Surg [Am] 1981 ;63-A:1472-81.

Friedlaender GE. Current concepts review : bone-banking. J Bone Joint Surg $[\mathrm{Am}] 1982 ; 64-\mathrm{A}: 307-11$.

Friedlaender GE, Mankin HJ. Bone banking: current methods and suggested guidelines. Am Acad Orthop Surg Instr Course Lect $1981 ; 3: 36-55$.

Friedlaender GE, Strong DM, Sell KW. Studies on the antigenicity of bone: 1 . Freeze dried and deep-frozen bone allografts in rabbits. $J$ Bone Joint Surg [Am] 1976;58-A :854-8.

Goldstein LA. Treatment of idiopathic scoliosis by Harrington instrumentation and fusion with fresh autogenous iliac bone grafts: results in eighty patients. J Bone Joint Surg [Am] 1969;51-A:209-22.

Johnson CA, Brown BA, Lasky LC. Rh immunization caused by osseous allograft. (Letter.) $N$ Engl J Med 1985;312:121-2.

Langer F, Czitrom A, Pritzker KP, Gross AE. The immunogenicity of fresh and frozen allogenic bone. J Bone Joint Surg [Am] 1975;57-A:216-20.

Malinin TI, Brown MD. Bone allografts in spinal surgery. Clin Orthop $1981 ; 154: 68-73$.

Malinin TI, Rosomoff HL, Sutton CH. Human cadaver femoral head homografts for anterior cervical spine fusions. Surg Neurol 1977;7:249-51.

McCarthy RE, Peek RD, Morrissy RT, Hough AJ Jr. Allograft bone in spinal fusion for paralytic scoliosis. J Bone Joint Surg [Am] 1986;68-A :370-5.

Stabler CL, Eismont FJ, Brown MD, Green BA, Malinin TI. Failure of posterior cervical fusions using cadaveric bone graft in children. $J$ Bone Joint Surg [Am] 1985;67-A:370-5.

Tomford WW, Ploetz JE, Mankin HJ. Bone allografts of femoral heads: procurement and storage. J Bone Joint Surg [Am] 1968;68-A :534-7.

Tomford WW, Starkweather RJ, Goldman MH. A study of the clinical incidence of infection in the use of banked allograft bone. $J$ Bone Joint Surg [Am] $1981 ; 63-\mathrm{A}: 244-8$. 